\title{
Um Sistema Inteligente para a Avaliação de Risco da DRC e Encaminhamento de Pacientes em Emergência para Unidades de Saúde
}

\author{
Andressa Carvalho Melo da Silveira ${ }^{1}$, Leandro Dias da Silva ${ }^{1}$, Álvaro Sobrinho ${ }^{1,2}$ \\ ${ }^{1}$ Instituto de Computação - Universidade Federal de Alagoas (UFAL) \\ Maceió - AL - Brasil \\ ${ }^{2}$ Instituto de Computação - Universidade Federal do Agreste de Pernambuco (UFAPE) \\ Garanhuns - PE - Brasil \\ acmsq@ic.ufal.br, leandrodias@ic.ufal.br, alvaro.alvares@ic.ufal.br
}

\begin{abstract}
The high incidence and prevalence of Chronic Kidney Disease (CKD), often caused by late diagnoses, is a critical public health problem. Qualitative and quantitative comparative analyzes were performed, by a systematic literature review and an experiment with machine learning techniques, respectively. The J48 decision tree, with $95.00 \%$ accuracy, was used to develop an intelligent system to assess the risk of CKD. In addition, when the patient with CKD is out of his/her municipality and an emergency occurs, the system recommends the patient to attend to an appropriate healthcare facility, depending on the clinical situation, to prevent the late or inadequate healthcare.
\end{abstract}

Resumo. A alta incidência e prevalência de Doença Renal Crônica (DRC), frequentemente causada por diagnósticos tardios, é um problema crítico de saúde pública. Análises comparativas qualitativas e quantitativas foram realizadas usando uma revisão sistemática da literatura e um experimento com técnicas de aprendizado de máquina, respectivamente. A árvore de decisão J48, com 95,00\% de acurácia, foi usada para desenvolver um sistema inteligente para avaliar o risco de DRC. Além disso, quando o paciente com DRC está fora de seu município e ocorre uma emergência, o sistema recomenda que o paciente compareça a uma unidade de saúde apropriada, dependendo da situação clínica, para evitar cuidados de saúde tardios ou inadequados.

\section{Introdução}

A Doença Renal Crônica (DRC) é um problema de saúde pública mundial caracterizado por dano renal permanente [Webster et al., 2017]. Por se tratar de uma doença assintomática, a DRC costuma ser diagnosticada quando o paciente encontra-se nos últimos estágios da doença. O diagnóstico tardio é um problema, especialmente em países em desenvolvimento como o Brasil, que geralmente sofrem com a precariedade da atenção primária à saúde em locais de difícil acesso, tais como zonas rurais.

Com o intuito de fornecer recursos para auxiliar os usuários a realizarem o automonitoramento de risco de DRC em países em desenvolvimento, Sobrinho et al. [2018] apresentaram um protótipo em Android de um sistema denominado MultCare. O sistema MultCare foi desenvolvido baseado em diretrizes médicas, especificações formais, 
avaliação de eficácia e testes de usabilidade. Neste trabalho, resultados obtidos por Sobrinho et al. [2018] são estendidos com o objetivo de aumentar a confiança em avaliações de risco da DRC, melhorar usabilidade e adicionar novas funcionalidades.

O estudo foi guiado por duas Questões de Pesquisa Principais (QPP): (1) qual é o classificador baseado em Aprendizado de Máquina (AM) mais adequado para o diagnóstico de DRC em países em desenvolvimento? e (2) quais são os atributos mais adequados para o diagnóstico de DRC em países em desenvolvimento? O objetivo principal com este trabalho foi utilizar AM no desenvolvimento de um sistema Web para auxiliar o monitoramento da DRC em países em desenvolvimento.

As principais contribuições com este trabalho incluem: (1) resultados de uma análise comparativa sobre técnicas de AM para o diagnóstico da DRC em países em desenvolvimento; (2) disponibilização de um sistema Web para a identificação e monitoramento da DRC usando AM; e (3) disponibilização de um sistema Web para o encaminhamento de pacientes em situações de emergência. Os resultados da análise comparativa foram publicados no periódico IEEE Access [Sobrinho et al., 2020], já os dois software foram registrados pelo Instituto Nacional da Propriedade Industrial (INPI): (1) MultCare Paciente $^{1}$ e (2) MultCare Triagem ${ }^{2}$.

\section{Metodologia}

Para contemplar os objetivos mencionados anteriormente, nessa sessão é apresentada a metodologia utilizada para o desenvolvimento de uma Revisão Sistemática da Literatura (RSL) focada no diagnóstico de DRC auxiliado por AM, um experimento baseado em técnicas de AM e o desenvolvimento de um sistema Web.

\subsection{Protocolo da Revisão Sistemática da Literatura}

O protocolo da RSL foi estruturado com base nas diretrizes de RSL em engenharia de software propostas por Kichenham and Charters (2007) e Wohlin [2014]. As bases de dados IEEE Xplore Library, ACM Digital Library, Scopus e PubMed foram utilizadas para realizar pesquisas com base em critérios gerais de seleção e critérios de inclusão e exclusão. Para melhorar a qualidade da pesquisa, a RSL foi realizada também utilizando a técnica snowballing e buscas manuais no Google Scholar@. O objetivo principal com a RSL foi responder as duas QPP deste trabalho.

\subsection{Aprendizado de Máquina}

Para o experimento foi considerado um conjunto de dados contendo indivíduos diagnosticados ou não com DRC (60 prontuários médicos). Os prontuários foram avaliados por uma nefrologista experiente e dois nefrologistas menos experientes. Os nefrologistas classificaram os sujeitos considerando as classes de baixo risco, risco moderado, alto risco e risco muito alto, de acordo com a diretriz KDIGO CKD [Lamb et al., 2013] e os atributos disponíveis. Portanto, trata-se de um problema de classificação multiclasse (desbalanceadas) usando uma quantidade reduzida de dados.

Os atributos do conjunto de dados foram escolhidos principalmente com base em diretrizes médicas amplamente aceitas pela comunidade geral de nefrologistas [Lamb

\footnotetext{
${ }^{1}$ Processo $N^{\mathrm{o}}$ : BR512020002085-2

${ }^{2}$ Processo No: BR512020002084-4
} 
et al., 2013; Forbes and Gallagher, 2020; Inker et al., 2014]. O conjunto final de atributos da DRC com foco em países em desenvolvimento incluiu hipertensão, Diabetes Mellitus (DM), creatinina, uréia, albuminúria, idade, sexo e Taxa de Filtração Glomerular (TFG). Os classificadores baseados em AM foram selecionados com base em um subconjunto de técnicas identificadas na RSL: Random Forest (RF), Naive Bayes (NB), Support Vector Machine (SVM), Multilayer Perceptron (MLP), K-Nearest Neighbor (KNN) e árvore de decisão J48.

\subsection{Desenvolvimento Web}

Com base na análise comparativa, um sistema Web inteligende de suporte à decisão clínica, denominado MultCare Paciente, foi desenvolvido. O back-end do sistema foi implementado, principalmente, usando a linguagem de programação Java, serviços Web e as interfaces de programação de aplicação Weka $\bigodot^{3}$ e Drools $\bigodot^{4}$. O front-end do sistema foi implementado usando, principalmente, HTML 5, Bootstrap, JavaScript e Vue.js.

\section{Resultados e Discussão}

Nesta sessão são apresentados os resultados relacionados com a análise comparativa [Sobrinho et al., 2020].

\subsection{Revisão Sistemática da Literatura}

Com base nos resultados da RSL, os estudos selecionados apresentaram algumas limitações, que indicam pelo menos uma lacuna de pesquisa: falta de análise comparativa dos classificadores baseados em AM considerando características específicas de países em desenvolvimento, como, por exemplo, altos níveis de pobreza. Em nenhum dos trabalhos selecionados foram consideradas as implicações de características específicas enfrentadas para realizar um diagnóstico completo da DRC em países em desenvolvimento.

Entre os 27 estudos aceitos na RSL, 13 estudos utilizaram algum tipo de rede neural, seguido de SVM com 12 estudos; NB foi usado em 8 estudos; técnicas baseadas em árvore em 7 estudos; KNN em 6 estudos; e AM baseado em conjunto e regressão logística em 4 estudos. Os resultados da RSL indicaram que a escolha do classificador baseado em AM e dos atributos da DRC dependem do contexto da aplicação. A análise dos resultados de classificação durante a RSL demonstraram que, geralmente, um maior número de atributos alcança um melhor desempenho para os classificadores baseados em AM. Entretanto, a necessidade do uso de vários biomarcadores da DRC na realização de classificações aumenta o custo para os usuários que se beneficiam de diagnósticos auxiliados por computador. O alto custo é um problema crítico para países em desenvolvimento, que enfrentam altas taxas de pobreza.

\subsection{Experimento com Aprendizado de Máquina}

O experimento foi focado no diagnóstico da DRC em países em desenvolvimento para melhorar a qualidade da análise comparativa e solucionar a lacuna de pesquisa identificada na RSL. O método $k$-fold Cross-Validation (com $\mathrm{k}=10$ ) foi usado para avaliar os classificadores com base em 114 registros, incluindo os 60 dados de prontuários de

\footnotetext{
${ }^{3}$ https://www.cs.waikato.ac.nz/ml/weka/

${ }^{4}$ https://www.drools.org/
} 
pacientes do hospital universitário da UFAL e 54 aumentados. O aumento de dados foi realizado apenas no conjunto de treinamento para cada uma das 10 dobras. As amostras foram aumentadas duplicando os registros de prontuários, modificando cuidadosamente os atributos (aumentando cada biomarcador da DRC em 0,5). A validade dos dados aumentados foi verificada por uma nefrologista com mais de 30 anos de experiência no tratamento e diagnóstico da DRC, que analisou cada registro quanto à classificação de risco adequada.

Considerando a QPP.1 os resultados obtidos pelo experimento sugerem o J48 e a RF como os mais adequados. Os classificadores apresentaram 95,00\% e 93,33\% de acurácia para o conjunto de dados da DRC coletado para o experimento, seguido por NB com $88,33 \%$, SVM com 76,66\%, MLP com 75,00\% e KNN com 71,67\%. Na Tabela 1 são apresentados os resultados do valor médio das métricas de desempenho.

Tabela 1. Resultados da avaliação de qualidade para os classificadores e o nefrologista mais experiente usando as métricas de desempenho. ${ }^{5}$

\begin{tabular}{c|r|r|r|r|r|r}
\hline & \multicolumn{1}{|c|}{ J48 } & \multicolumn{1}{c|}{ RF } & \multicolumn{1}{c|}{ NB } & SVM & MLP & KNN \\
\hline CCI (\%) & 95.00 & 93.33 & 88.33 & 76.66 & 75.00 & 71.67 \\
\hline ICI (\%) & 5.00 & 6.67 & 11.67 & 23.33 & 25.00 & 28.33 \\
\hline TP & 0.95 & 0.93 & 0.88 & 0.77 & 0.75 & 0.72 \\
\hline FP & 0.01 & 0.01 & 0.05 & 0.22 & 0.28 & 0.16 \\
\hline P & 0.97 & 0.99 & 0.95 & 0.78 & 0.78 & 0.79 \\
\hline ROC & 0.96 & 0.99 & 0.97 & 0.79 & 0.83 & 0.88 \\
\hline PRC & 0.94 & 0.97 & 0.96 & 0.67 & 0.82 & 0.86 \\
\hline
\end{tabular}

Além disso, os resultados das classificações foram comparados com a opinião de um nefrologista experiente com os classificadores baseados em AM usando a estatística kappa de Cohen. SVM, MLP e KNN apresentaram concordância moderada com k = $0,5807, \mathrm{k}=0,5418$ e $\mathrm{k}=0,5112$, respectivamente; seguido por $\mathrm{NB}(0,8170), \mathrm{RF}(\mathrm{k}=$ $0,8950)$ e J48 $(\mathrm{k}=0,9221)$, que apresentaram concordância quase perfeita com o nefrologista experiente.

Para melhorar a discussão, os resultados dos classificadores de AM, treinados com base na opinião do nefrologista mais experiente, foram comparados à opinião de dois nefrologistas menos experientes usando a estatística kappa de Cohen. O desempenho dos classificadores diminuiu, apresentando concordância moderada com os dois nefrologistas. Também foi realizada a comparação completa, considerando os resultados dos classificadores e as opiniões dos três nefrologistas, onde a concordância continuou moderada. A discordância entre os três nefrologistas, indica a relevância do uso de soluções para o diagnóstico precoce da DRC auxiliado por computador em países em desenvolvimento para auxiliar nefrologistas menos experientes em tomadas de decisões clínicas.

Portanto, são consideradas duas possibilidades: (1) RF junto com resultados interpretados ou (2) árvore de decisão J48. O classificador J48 usou apenas 4 atributos para classificar o risco (DM, TFG, albuminúria e idade), exigindo 1 exame de sangue e

\footnotetext{
${ }^{5}$ Correctly Classified Instances (CCI), Incorrectly Classified Instances (ICI), True positive (TP) rate, False positive (FP) rate, Precision (P), Receiver operating characteristic (ROC) area e Precision-recall curve $(P R C)$ area
} 
1 exame de urina quando a DM já está avaliada. Além disso, apresentar o resultado da avaliação como uma árvore de decisão pode simplificar o diagnóstico final de um médico de atenção primária.

\subsection{Sistema Web}

A versão Web do sistema foi desenvolvida para aprimorar a análise de risco da DRC usando o classificador J48. As principais funcionalidades implementadas ${ }^{6}$ incluem: (1) controle de acesso; (2) gerenciamento de exames, alergias e medicamentos; (3) realização de análise de risco; (4) monitoramento de DM e hipertensão; e (5) encaminhamento em situações de emergência.

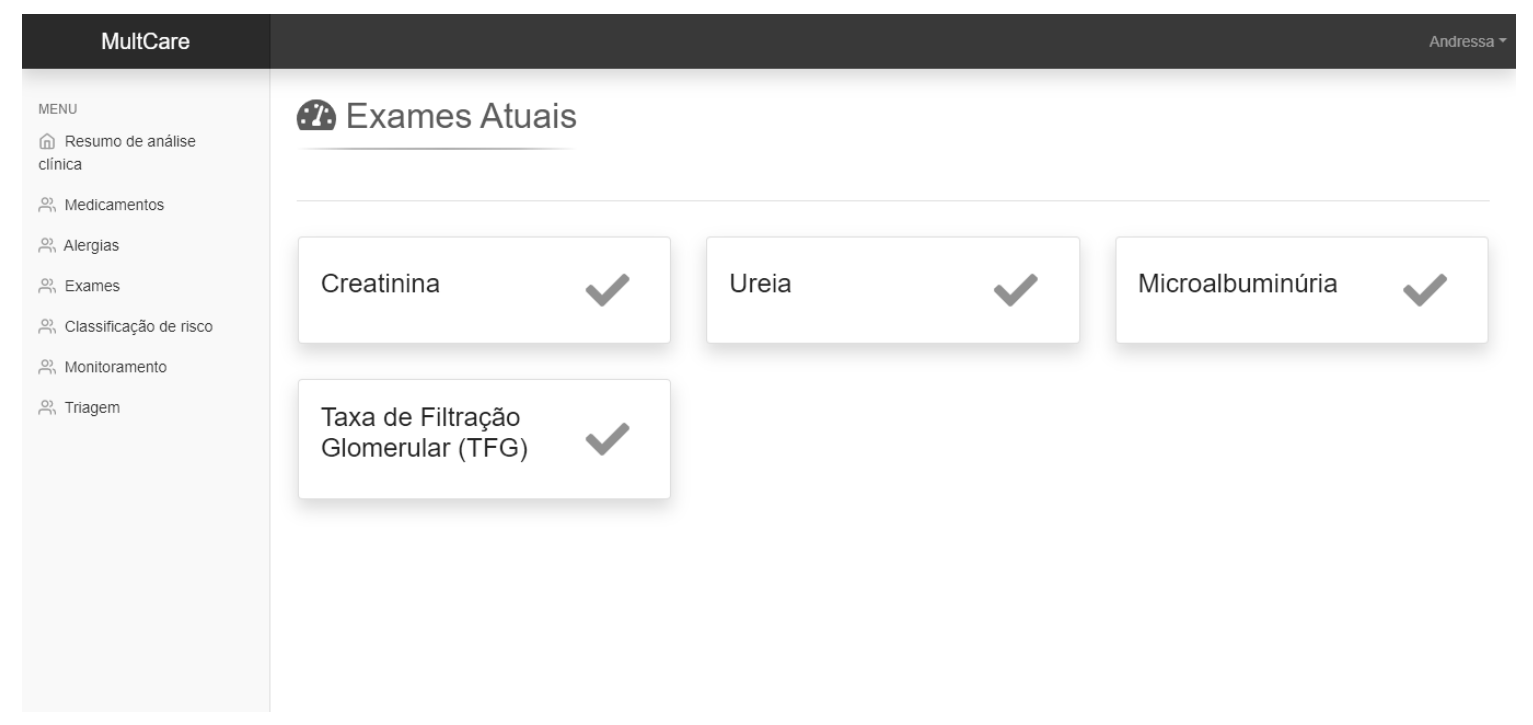

Figura 1. Principal interface gráfica do usuário.

Na Figura 1 é ilustrado um exemplo de interface gráfica de usuário do sistema.O conjunto completo de dados da DRC, usado durante a análise comparativa, foi considerado no treinamento do classificador J48. A interface de programação de aplicação do Weka@ foi usada na implementação do sistema.

Nos casos de risco moderado, risco alto ou risco muito alto, um documento clínico, estruturado usando o padrão HL7 Clinical Document Architecture (CDA), é enviado por e-mail ao médico responsável pelo monitoramento do paciente. O CDA é um arquivo XML que contém os dados da análise de risco, uma árvore de decisão da análise de risco e o registro pessoal do paciente. Em caso de baixo risco, o sistema registra apenas os resultados da análise de risco para acompanhar a situação clínica do paciente e não envia o alerta ao médico.

Como outra contribuição foi desenvolvido um sistema baseado em conhecimento (como parte do MultCare Paciente), usando a ferramenta Drools@, para encaminhar o paciente com DRC e fatores de risco a um serviço de saúde adequado em uma situação de emergência. Neste recurso é considerado o cenário em que o paciente está fora de sua cidade e não conhece as instalações corretas para receber tratamento, de acordo com a sua situação atual de saúde. As seguintes patologias foram definidas para o projeto do

\footnotetext{
${ }^{6}$ Principais funcionalidades: youtu.be/icdpVJuUw00 e youtu.be/nuyGMpEdNOs.
} 
sistema especialista: hiperglicemia, hipoglicemia, hipercalemia e hipocalemia. Para estas patologias, o paciente pode ser encaminhado para unidades de pronto atendimento ou emergências de hospitais, variando com base na situação de saúde atual do paciente.

A base de conhecimento do sistema foi projetada com base em informações obtidas por meio de diretrizes médicas (e.g., diretrizes da sociedade brasileira de diabetes [Diabetes, 2000]), entrevista com um nefrologista experiente, condições clínicas atuais do paciente e outros fatores, como, por exemplo, o uso de algumas medicações, o álcool, se consumido em excesso, e a insulina utilizada no tratamento de diabetes.

\section{Conclusão}

No contexto de países em desenvolvimento, os custos decorrentes do uso de software para auxiliar no diagnóstico da DRC devem ser os mais baixos possíveis, especialmente em ambientes rurais e de difícil acesso. O número de atributos de DRC usados durante as classificações de risco afetam o custo de uso e o desempenho dos classificadores. A árvore de decisão J48 usou apenas 4 atributos para classificar o risco (DM, TFG, albuminuria e idade), apresentando concordância quase perfeita com um nefrologista experiente. Como trabalhos futuros, tem-se: (1) a ampliação para outras patologias relacionadas com a DRC; (2) realizar testes de aplicabilidade clinica com especialistas na área da nefrologia; e (3) realizar testes de usabilidade.

\section{Referências}

S. B. Diabetes. Diretrizes da sociedade brasileira de diabetes. Grupo Gen-AC Farmacêutica, 2000.

A. Forbes and H. Gallagher. Chronic kidney disease in adults: assessment and management. Clinical Medicine, 20(2):128, 2020.

L. A. Inker, B. C. Astor, C. H. Fox, T. Isakova, J. P. Lash, C. A. Peralta, M. K. Tamura, and H. I. Feldman. Kdoqi us commentary on the 2012 kdigo clinical practice guideline for the evaluation and management of ckd. American Journal of Kidney Diseases, 63 (5):713-735, 2014.

B. Kitchenham and S. Charters. Guidelines for performing systematic literature reviews in software engineering. Technical report, 2007.

E. J. Lamb, A. S. Levey, and P. E. Stevens. The kidney disease improving global outcomes (kdigo) guideline update for chronic kidney disease: evolution not revolution. Clinical chemistry, 59(3):462-465, 2013.

A. Sobrinho, L. D. da Silva, A. Perkusich, M. E. Pinheiro, and P. Cunha. Design and evaluation of a mobile application to assist the self-monitoring of the chronic kidney disease in developing countries. BMC Medical Informatics and Decision Making, 18 (1):7, Jan 2018. ISSN 1472-6947. doi: 10.1186/s12911-018-0587-9.

A. Sobrinho, A. C. D. S. Queiroz, L. D. Da Silva, E. D. B. Costa, M. E. Pinheiro, and A. Perkusich. Computer-aided diagnosis of chronic kidney disease in developing countries: A comparative analysis of machine learning techniques. IEEE Access, 8:2540725419, 2020.

A. C. Webster, E. V. Nagler, R. L. Morton, and P. Masson. Chronic kidney disease. The lancet, 389(10075):1238-1252, 2017.

C. Wohlin. Guidelines for snowballing in systematic literature studies and a replication in software engineering. In Proceedings of the 18th international conference on evaluation and assessment in software engineering, pages 1-10, 2014. 